\title{
A Survey on Green Cloud Computing
}

\author{
Sonia Panwar \\ Student, M.Tech(CSE), \\ Deenbandhu Chhotu Ram University, Murthal.
}

\author{
Kavita Rathi \\ Assistant Professor, CSE \\ Deenbandhu Chhotu Ram University, Murthal.
}

\begin{abstract}
Network based cloud computing is an alternative to officebased computing. As cloud computing is expanding day by day, energy consumption within cloud is also growing. So there is a need for the management of energy consumption in technology (information and communication) section. Data centers have received more attention in case of energy consumption. This paper contains study on different scheduling approaches on cloud computing.
\end{abstract}

\section{Keywords}

Cloud computing, Green computing, Data centers, Energy consumption

\section{INTRODUCTION}

Delivery of network-based services is enabled due to the highly availability of IP sector and high-speed internet [1]. These network-based services has expanded to storage and computing. Which are offered to corporate and individual users? Such services are called "cloud computing".

Cloud computing is a convenient model for enabling virtual (on-demand) network access for sharing computing resources which are configurable and available with minimum management effort. [1]

The most significant part of ":Green" concept is Green Cloud Computing which will helpful in reduction of green house gases which will automatically helpful in reduction of global warming [1]. A cloud can be private or public:

A Private Cloud: A cloud which is available only within an enterprise and can be used by its enterprise only. Private cloud is very exclusive and only limited participants can access it. These are significant for large organization.

A Public Cloud: A Public cloud is designed in such a way that it can be used by any user with an internet connection and provides a similar range of services and capabilities.

Cloud computing can be energy saving if the user migrates to use computer which consume lower energy. The power consumption management in data centers has a number of improvements in energy efficiency. It is precious to understand how the energy consumption in data centers which host cloud computing can be minimize. A better analysis is required for the analysis of total energy consumption in cloud computing.

\section{CLOUD SERVICE MODELS}

We focus on three cloud service models-
1) Software as a service
2) Storage as a service
3) Processing as a service

\subsection{Software as a service}

In case of Software as a service, latest version of any software as per requirement of client can be provided and annual or monthly fee can be charged for the access of that software. Additionally, this software is available or hosted on the cloud and all computational tasks are performed in the cloud [1]. The user can only transmit command and computation is done in cloud and receives results.

\subsection{Storage as a service}

In this service, client's data can be stored in the cloud. All computation is performed on client's PC. Data stored in cloud may be document, photos, and videos. Any computer which is connected with internet can access files stored in cloud.

\subsection{Processing as a service}

This service facilitate user with a lot of powerful resources of server for computational tasks. Computing task of high demand are uploaded and processed on the server and computing results are equipment, storage function and processing model in data centers [1].

\section{ISSUES WITH CLOUD}

The main concept of cloud system is to avail the services and the resources to the distributed users. These services are provided by different service providers that are integrated to the system to avail the services to different users. The cloud system concept is beneficial to the user economically so that better adaptation to the services will be done by the environment. Depending on the type of services, the provider to the system is defined under the layered environment. These layers are described in the earlier section, But when a client perform the service request all there service layers get activated in an integrated way. One of the important issues in cloud environment is its complex architecture. As the model is supported by web access so that it require the complex architecture to provide the effective deployment of the service and to provide the virtual environment to the system.

\section{WHAT IS GREEN CLOUD COMPUTING?}

As energy is limited and global climate is continuously changing it leads our concern to the energy consumption if data centers. Green cloud computing is energy efficient computing. Green cloud computing provide a solution so that it can minimize operational costs as well as reduce the environmental impact [2]. Green computing can reduce the $\mathrm{CO} 2$ emission where client can use the web services to demand the resources and pay for resources that are actually consumed.

Virtualization is main technique which abstracts the resources (both hardware and software) and also reduces total number of hardware equipments. To manage the energy consumption "green virtual network" must built in cloud computing. In cloud computing an abstraction is provided by network virtualization for network simplifying resource provisions [3]. 


\section{GREEN CLOUD COMPUING ARCHITECTURE}

In this architecture, the user resides at top level that will perform the service or the cloud request. Actually the cloud system is designed to satisfy the end user or the customer. The end user can be a single user of the organization. The cloud server specification defined by the user is analyzed under different vectors. User can select the service required from the cloud server as well as user can also decide the particular cloud server from which the service will be retrieved. A cloud user can exist in different cloud environment such as public environment, private environment or the community environment. With each level, the cost specification, security specification is differ [4][5].
The second level of cloud architecture is the application level interface. It is the actually the layer, where the application requirement of cloud is defined. This layer is defined as the cloud to user interface.

The physical layer or lower level of cloud architecture is represented by the cloud server itself. This layer contains the database layer integrated with virtual cloud. This layer is responsible to perform the actual service allocation and the execution. The cloud system defined at this level is most complex respective to the user. As the cloud system is present in web form, it is more complex but provide effective services.

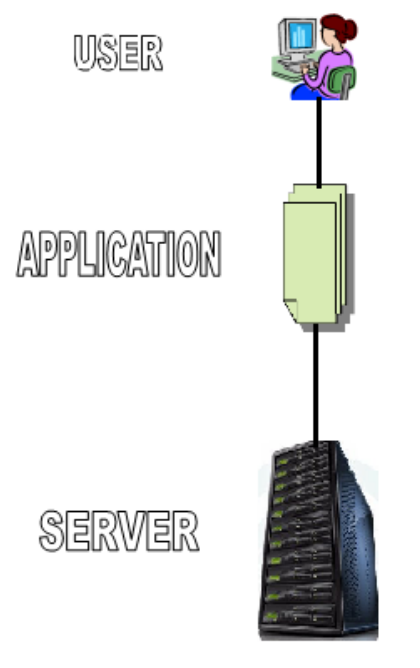

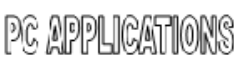

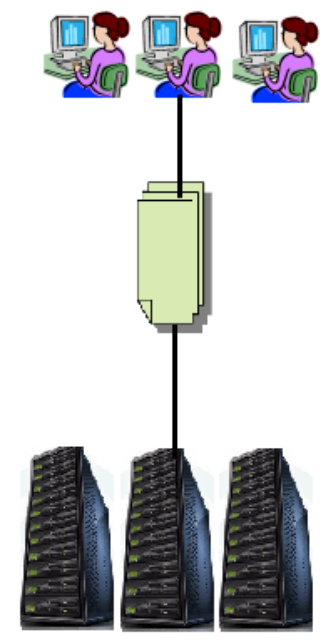

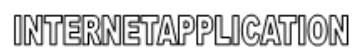

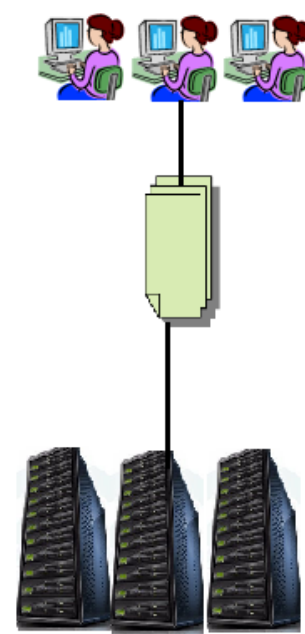

GேOVD APPLIGATION

Fig 1: Cloud computing architecture

\section{HOW TO MEASURE ENERGY CONSUMPTION}

Its not possible to manage anything if there are not possible standards for its measurement. There must have some standards to measure the energy consumption. Energy-use metrics is an IT standard for energy consumption measurement. There are also the measurements for green buildings such as:

\subsection{LEED}

Green Building Rating System known as the Leadership in Energy and Environmental design (LEED) was developed by USGBC (the U.S. Green Building Council) [6].

\subsection{EPEAT}

There is another metric for the use of green IT which is EPEAT (The Electronic Product Environmental Assessment Tool) which was created by IEEE (Institute of Electrical and Electronics Engineers). President BUSH signed an order in January 2007 due to which EPEAT got a boost according to it every electronic product which procured by federal agencies must meet the EPEAT standards.

\subsection{EPA}

All metrics which are useful in any aspect of data center, EPA is pushing must be a guideline for them. Or we can say that EPA is trying to make all the data centers to be GREEN [7].

\subsection{SPEC and PUE}

There are some other metrics which are specially used to measure energy efficiency in data centers and these are SPEC and PUE. SPEC is being developed by the EPA. PUE (Power Usage Effectiveness) is also used to measure energy efficiency for the data centers. Overall and high-density zone are the two measures of PUE.

\section{ENERGY CONSUMPTION MODELS}

This section describes energy consumption of computing and transport equipment over which cloud computing currently work. Energy consumption models are used to measure energy consumption per bit for storage, transport and processing meas. Energy consumption can be measured with the help of two fundamental measures i.e. storage per bit and energy per bit [8].

A cloud's energy efficiency can be measured as energy consumed by a bit of data which is processed during cloud computing [8]. 
An important issue while measuring energy consumption is the energy required in cooling and another overheads. The power usage efficiency (PUE) can be defined as the ratio of power consumption by a facility like data or switching center to the power

Consumption the IT equipment i.e. servers, storage, routers, etc. [1][8].

\subsection{User Equipment}

A user uses various devices while accessing cloud like mobile, PC, laptop etc. A computer consists of CPU, RAM, HDD, motherboard, peripheral devices, a power supply unit. Table 1 shows commonly used computer types that a user can use for PC and the energy consumed by these computers and power required by a 2.5" HDD. A terminal accepts commands from the clients or users side and starts communication with the server with the help of IP connection [8].

Table 1 Hardware in model of User Equipment

\begin{tabular}{|c|c|c|c|}
\hline & & $\begin{array}{l}\text { Power } \\
\text { Consur }\end{array}$ & tion \\
\hline Equipment & Parts & Idle & $\begin{array}{l}\text { Full } \\
\text { Load }\end{array}$ \\
\hline $\begin{array}{l}\text { Modern mid- } \\
\text { range computer }\end{array}$ & $\begin{array}{l}\text { Intel E5200 @ } 2.5 \\
\text { GHz, } 2 \text { GB RAM }\end{array}$ & $75 \mathrm{~W}$ & $130 \mathrm{~W}$ \\
\hline $\begin{array}{l}\text { Old mid-range } \\
\text { computer }\end{array}$ & $\begin{array}{lcr}\text { Intel } & \text { Pentium } & 4 \text { @ } \\
2.86 & \mathrm{GHz}, & 1 \mathrm{~GB} \\
\text { RAM } & & \end{array}$ & $135 \mathrm{~W}$ & $230 \mathrm{~W}$ \\
\hline $\begin{array}{l}\text { Modern high- } \\
\text { range computer }\end{array}$ & $\begin{array}{l}\text { Intel Q6600 @ } 2.4 \\
\text { GHz, 4 GB RAM }\end{array}$ & $120 \mathrm{~W}$ & $165 \mathrm{~W}$ \\
\hline Low-end laptop & $\begin{array}{l}\text { Intel Atom @ } 1.6 \\
\text { GHz, } 2 \text { GB RAM }\end{array}$ & $20 \mathrm{~W}$ & $20 \mathrm{~W}$ \\
\hline
\end{tabular}

\subsection{Data Centers}

Server, data storage, and a local area network are the main components of modern data center [1][8]. These data centers are connected to the rest of the network through the routers. Storage in the data centers can be provided by HDD. The storage space is consolidated and HDD used is centrally coordinated. Most of the organizations are moving towards green data centers which are implementation of IT data center devices and these IT devices are client, data storage, and servers.

Data centers require large amount of energy per square foot as compare to any other section of a building [2]. The server's capacity or performance is mainly depends on the task that is being performed, together with the operations, parallel processing and memory access.

In the data center a LAN aggregates the data or resource traffic from servers side into higher capacity (typically $10 \mathrm{GE}$ ) links and connects to the network core through a gateway router [8][10].

\subsection{Networks:}

This section contains a description of corporate \& internet network (IP) and the equipment use in those networks. Table 3 contains equipments used in calculating energy consumption in corporate and internet network (IP) and also the power consumption and capacity of this equipment.
Table 2 Equipment in model of Network

\begin{tabular}{|c|c|c|c|}
\hline & Equipment & Capacity & $\begin{array}{l}\text { Power } \\
\text { Consumption }\end{array}$ \\
\hline $\begin{array}{l}\text { Ethernet } \\
\text { Switch } \\
\text { (Small) }\end{array}$ & Cisco 4503 & $65 \mathrm{~Gb} / \mathrm{s}[41]$ & $474 \mathrm{~W}$ [41] \\
\hline $\begin{array}{l}\text { Ethernet } \\
\text { Switch }\end{array}$ & Cisco 6509 & $160 \mathrm{~Gb} / \mathrm{s}[40]$ & $3.6 \mathrm{~kW}[40]$ \\
\hline BNG & Juniper E320 & $64 \mathrm{~Gb} / \mathrm{s}[40]$ & $3.4 \mathrm{~kW}[40]$ \\
\hline $\begin{array}{l}\text { Provider } \\
\text { Edge }\end{array}$ & Cisco 12816 & $\begin{array}{l}162 \quad \mathrm{~Gb} / \mathrm{s} \\
{[41]}\end{array}$ & $4.24 \mathrm{~kW}[41]$ \\
\hline Core router & Cisco CRS-1 & $\begin{array}{l}642 \quad \mathrm{~Gb} / \mathrm{s} \\
{[40]}\end{array}$ & $10.7 \mathrm{~kW}[40]$ \\
\hline $\begin{array}{l}\text { WDM } \quad(800 \\
\text { km) }\end{array}$ & Fujitsu 7700 & $42 \mathrm{~Gb} / \mathrm{s}$ [44] & $\begin{array}{ll}136 & \text { W/channel } \\
{[21]} & \end{array}$ \\
\hline
\end{tabular}

\section{SCHEDULING}

Scheduling is considered as the arrangement of the requests performed by different users in an organized and defined form. This form is defined under the real time scenario and able to improve the system capability. The cloud system defined under various process and memory based constraints is able to provide the generalized system allocation to the environment. There are number of scheduling and queuing approaches so that the multiprogramming based shared system is provided [14][15][16].

\subsection{Single Shared Ready Queue}

As a process is executed by the user, it is maintained in a queue called job queue, process queue or ready queue. It is the simplest form of job queue. As the name suggest, the traditional form of queue accept data from one end and dispatch data from second end. The presented queue based system is shared by number of processor. These processor system are shares the single shared queue. The processor systems are here described as the virtual machine present in same cloud environment. Now if the processor is not busy and demands for a process for the execution one process is taken out from the job queue and available to the processor. In the simplest form of scheduling First Come First Server (FCFS) approach is implemented in such system, where the order of process request by the user is taken by the cloud to execute it on server. In the second form of system, the SJF is defined and implemented. In such system, the processor utilization is taken as the decision vector. The process that requires the shortest time span on the processor will be executed first [15][16].

\subsection{Co scheduling}

Co-Scheduling is the common execution policy applied on multiple processes to run simultaneously on different processors. Scheduling is very common cloud environment and provides the integration with processing unit so that the cooperative communication will be obtained. These processes are executed without any cooperation. Multiple processors to the system provide the parallel execution of the task on multiple processors so that the execution latency on the system will be reduced. The system is able to perform the process execution in an effective way. The scheduling process 
in the environment so that cooperative scheduling is the foremost requirement in distributed environment.

\subsection{Static Co scheduling}

The static co scheduling defines the scheduling process in the integrated environment. In which a global queue is present so that single job list is maintained to schedule the jobs in the cloud environment. These jobs are defined in an order so that effective context switching over the processors will be done to check the availability of the processors. There is the master scheduler that will perform the analysis on the processors and identify the best processor for the current job. This global scheduler moves between the processor the analysis.

\subsection{Local Co scheduling}

Another kind of scheduling system adapted by parallel processor system is the local co-scheduling system. This kind of system are used by the processor system in an independently schedule processes so that effective allocation of the processes to the processor will be done. This kind of systems degrades the system performance significantly because the scheduling is not coordinated across the multiple processors. The architecture of such systems seems easy but it gives the unequal load distribution.

\subsection{Implicit Co-scheduling}

Another scheduling approach adapted in a cloud system is implicit co-scheduling approach. This approach is also called local scheduling approach. According to this approach each processor is having the local scheduler as well as their existing a global scheduler. Two level scheduler defined in the system defined the effective coverage to the system. This system provides the high level scheduling on the main scheduler and low level scheduling on the local scheduler. The behavior analysis on each scheduling system is done so that decision making will be done reliably.

\section{CONCLUSION}

In this paper, we have presented a literature survey on cloud computing and various issues associated with it and an analysis of energy consumption in cloud computing which considered both public and private clouds. It also presents energy consumption due to data processing, data storage, switching and transmission, and how the green cloud computing is helpful in efficient energy consumption.

A little survey is also done on "How to measure energy consumption" and "various energy consumption models i.e. User Equipments, Data Centers and Networks".

Survey on scheduling shows various scheduling and queuing approaches so that the multiprogramming based shared system is provide i.e. Single Shared Ready Queue, Co scheduling, Static Co scheduling, Local Co scheduling, Implicit Co-scheduling.

\section{REFERENCES}

[1] Jayant Baliga, Robert W.A. Ayre, Kerry Hinon and Rodney S.Tucker, Fellow IEEEE," Green Cloud Computing: Balancing energy in processing, storage, and Transmission".

[2] Mark D. Ryan School of Computer Science, University of Birmingham, Birmingham B15 2TT, UK," Cloud computing security: The scientific challenge and a survey of solutions".

[3] Ruay-Shiung Chang and Chia-Ming Wu," Green Virtual Networks for Cloud Computing
[4] Kento Sato, "A Model-Based Algorithm for Optimizing I/O Intensive Applications in Clouds using VM-Based Migration", 9th IEEE/ACM International Symposium on Cluster Computing and the Grid 978-0-7695-3622-4/09@ 2009 IEEE

[5] Takahiro Hirofuchi, "Enabling Instantaneous Relocation of Virtual Machines with a Lightweight VMM Extension", 2010 10th IEEE/ACM International Conference on Cluster, Cloud and Grid Computing 9780-7695-4039-9/10@ 2010 IEEE

[6] John Lamb, Ph.d., Senior Technical Staff Member IBM " "Green IT and use of private Cloud Computing in South Africa".

[7] Truong Vinh Truong Duy and Yukinori Sato and Yasushi Inoguchi from Japan Advanced Institute of Science and Technology," Performance Evaluation of a Green Scheduling Algorithm for Energy Saving in Cloud Computing".

[8] Jayant Baliga, Robert W.A. Ayre, Kerry Hinon and Rodney S.Tucker, Fellow IEEEE," Green Cloud Computing: Balancing energy in processing, storage, and Transmission".

[9] Asif Qumer Gill and Deborch Bunker," An Empirical Analysis of Cloud, Mobile, Social and Green Computing".

[10] Jianxin Li, Bo Li, Tianyu Woa, Chunming Hu, Jinpeng Huai, Lu Liu, K.P. Lam," CyberGuarder: A virtualization security assurance architecture for green cloud computing".

[11] Chang Liu, Xuyun Zhang, Chi Yang, Jinjun Chen," Future Generation Computer Systems CCBKE Session key negotiation for fast and secure scheduling of scientific applications in cloud computing"

[12] Brian Dougherty, Jules White, Douglas C. Schmidt," Model-driven auto-scaling of green cloud computing infrastructure".

[13] Bruce Baikie and Dr. Laura Hosman.” Green Cloud Computing in Developing Regions".

[14] J. Brandt, "Using Cloud Constructs and Predictive Analysis to Enable Pre-Failure Process Migration in HPC Systems", 2010 10th IEEE/ACM International Conference on Cluster, Cloud and Grid Computing 9780-7695-4039-9/10@ 2010 IEEE.

[15] Sumit Kumar Bose, "CloudSpider: Combining Replication with Scheduling for Optimizing Live Migration of Virtual Machines Across Wide Area Networks", 2011 11th IEEE/ACM International Symposium on Cluster, Cloud and Grid Computing 978 0-7695-4395-6/11@ 2011 IEEE

[16] Shigeru Imai, "Elastic Scalable Cloud Computing Using Application-Level Migration", 2012 IEEE/ACM Fifth International Conference on Utility and Cloud Computing 978-0-7695-4862-3/12 @ 2012 IEEE

[17] Damien Borgetto, "Energy-efficient and SLA-Aware Management of IaaS Clouds", e-Energy 2012, May 9-11 2012, Madrid, Spain. ACM 978-1-4503-1055-0/12/05 